\title{
Correlação entre as restrições de participação em atividades de vida diária e o benefício do uso de próteses auditivas em adultos e idosos
}

\author{
Correlation between the handicap and the benefit of hearing \\ aid use in adults and elderly
}

\author{
Vívian Baptista da Luz¹, Monique Cantelli da Silvaㄹ, Renata Coelho Scharlach², Maria Cecília Martinelli Iórioº
}

\begin{abstract}
RESUMO
Objetivo: Estudar o benefício obtido com o uso de próteses auditivas em ambientes acusticamente favoráveis à comunicação, reverberantes, ruidosos e com sons aversivos e correlacioná-lo com as restrições de participação e limitações de atividades de adultos e idosos deficientes auditivos. Métodos: Foram avaliados 42 indivíduos atendidos na instituição em que o estudo foi realizado. Estes responderam aos questionários Hearing Handicap Inventory for Elderly ou for Adults e Abbreviated Profile of Hearing Aid Benefit, no momento da dispensação das próteses auditivas e após três meses de uso efetivo destas. Foram estudadas as restrições de participação, limitações auditivas e o benefício com o uso de próteses auditivas. Os resultados foram analisados estatisticamente. Resultados: Houve diferenças entre os escores dos questionários Hearing Handicap Inventory for Elderly e for Adults e entre os das subescalas Facilidade de Comunicação, Ambientes Reverberantes e Ruído Ambiental do questionário Abbreviated Profile of Hearing Aid Benefit, comparando as condições sem e com próteses auditivas. Houve correlação positiva entre o benefício mensurado na subescala Facilidade de Comunicação, nos idosos, e Ruído Ambiental, nos adultos, com a redução da restrição de participação. Conclusão: Houve redução das limitações de atividades e da restrição de participação em atividades de vida diária em adultos e idosos com o uso de próteses auditivas. Quanto maior o benefício obtido na subescala Facilidade de Comunicação em idosos e o benefício na subescala Ruído Ambiental nos adultos, maior a redução da restrição de participação.
\end{abstract}

Descritores: Reabilitação de deficientes auditivos; Auxiliares de audição; Perda auditiva neurossensorial; Audição; Adulto; Idoso

\section{INTRODUÇÃO}

A cada ano, a expectativa de vida se eleva. O avanço da idade vem acompanhado do envelhecimento dos sentidos, o que acarreta privações sensoriais, dentre elas a privação auditiva, denominada presbiacusia (perda auditiva neurossensorial).

A deficiência auditiva neurossensorial traz uma série de consequências, tais como problemas na compreensão da fala na presença e na ausência de ruído, isolamento, constrangimento, diminuição da participação social e prejuízo na interação familiar, prejudicando intensamente a qualidade de vida e a

Trabalho realizado no Departamento de Fonoaudiologia, Universidade Federal de São Paulo - UNIFESP - São Paulo (SP), Brasil.

(1) Programa de Pós-graduação (Mestrado) em Distúrbios da Comunicação Humana, Universidade Federal de São Paulo - UNIFESP - São Paulo (SP), Brasil.

(2) Departamento de Fonoaudiologia, Universidade Federal de São Paulo UNIFESP - São Paulo (SP), Brasil.

Endereço para correspondência: Vívian Baptista da Luz. R. Dr. Estácio Correia, 48/32, Boqueirão, Santos (SP), Brasil, CEP: 11045-020. E-mail: vividaluz@gmail.com

Recebido em: 24/11/2009; Aceito em: 22/8/2010 integração do indivíduo na sociedade.

As perdas auditivas neurossensoriais não atingem apenas os idosos. Este tipo de perda pode ocorrer também devido a traumas acústicos, ototoxicidade, meningite, etc; em qualquer fase da vida.

As habilidades auditivas e sociais prejudicadas em decorrência das perdas auditivas neurossensoriais são frequentemente descritas pela literatura, assim como também são descritos os benefícios que podem advir da utilização de uma prótese auditiva.

A avaliação clínica pressupõe o conhecimento e o uso de uma série de ferramentas técnicas assim como questões mais abrangentes relacionadas ao ser humano e suas relações e sentimentos sobre o meio que o cerca $^{(1)}$.

Os questionários de auto-avaliação são utilizados para avaliar o plano de intervenção fonoaudiológica no processo de adaptação das próteses auditivas e a efetividade da reabilitação ${ }^{(2-4)}$. A aplicação de tais questionários para avaliar o desempenho dos pacientes usuários de próteses auditivas e o benefício percebido por estes é de suma importância e recomendada ${ }^{(5-7)}$, visto que os testes realizados nas clínicas e 
consultórios não predizem a eficiência da prótese auditiva nas situações diárias de comunicação ${ }^{(5)}$.

$\mathrm{O}$ benefício do uso de próteses auditivas refere-se às vantagens obtidas pelos usuários com o uso da amplificação sonora $^{(8)}$, melhorando a qualidade de vida destes indivídu$\mathrm{os}^{(9)}$. O referido benefício pode ser mensurado por meio da comparação subjetiva das limitações e restrições vivenciadas nas situações com e sem próteses auditivas ${ }^{(10)}$, ou seja, das limitações de atividades (incapacidades) e das restrições de participação (também denominadas handicap auditivo), enfrentadas por deficientes auditivos, as quais se referem aos impactos da deficiência auditiva na participação social e nas atividades de vida diária.

Dentre os questionários de auto-avaliação mais utilizados na avaliação de resultados da intervenção fonoaudiológica por meio da adaptação de dispositivos eletrônicos, podem-se citar o Abbreviated Profile of Hearing Aid Benefit (APHAB) aplicado para avaliar as limitações de atividades decorrentes da deficiência auditiva; o Hearing Handicap Inventory for Elderly (HHIE), e o Hearing Handicap Inventory for Adults (HHIA), para avaliar as restrições de participação em idosos e adultos respectivamente; o Satisfaction With Amplification In Daily Life (SDAL) cujo objetivo é investigar a satisfação do usuário de próteses auditivas ${ }^{(11)}$ e o International Outcome Inventory for Hearing Aids (IOI-HA), que investiga um conjunto de sete itens da avaliação de resultados ${ }^{(12)}$, entre outros.

A hipótese do presente estudo foi a de que indivíduos adultos, ativos, desempenhando funções laborais e sociais, apresentam maior limitação e restrição de atividades do que os idosos, uma vez que existe uma solicitação maior do seu desempenho comunicativo nestas atividades. Por serem mais exigidos nas atividades de trabalho e sociais os adultos apresentariam maior percepção do benefício fornecido pela amplificação?

Tal hipótese e questionamento fundamentam a realização da presente pesquisa. Para cumprir com os objetivos propostos, os instrumentos selecionados foram os questionários de autoavaliação APHAB e o HHIE/HHIA.

O presente trabalho teve como objetivos avaliar de forma subjetiva as limitações auditivas e a restrição de participação em atividades de vida diária em adultos e idosos deficientes auditivos e estudar o benefício obtido com a utilização da prótese auditiva.

\section{MÉTODOS}

De acordo com as normas preconizadas para pesquisas utilizando seres humanos, o presente trabalho foi aprovado pelo Comitê de Ética em Pesquisa da Universidade Federal de São Paulo, sob o número 1389/07.

Para a realização deste trabalho, contou-se com a participação de 42 voluntários, selecionados de forma aleatória, os quais foram divididos em dois grupos amostrais após assinarem um Termo de Consentimento Livre e Esclarecido.

O primeiro grupo (Grupo Adultos - GA) foi composto por 17 indivíduos, seis homens e 11 mulheres, com idades entre 18 e 60 anos. O segundo grupo (Grupo Idosos - GI) foi composto por 27 idosos, dez homens e 17 mulheres, com idades iguais ou superiores a 60 anos. Todos os participantes da pesquisa eram pacientes da instituição em que o estudo foi realizado, portadores de perda auditiva neurossensorial bilateral, adquirida pós-lingual, de grau leve a moderadamente severo e não apresentaram alterações cognitivas evidentes. Ressalta-se que o estudo do benefício do uso das próteses auditivas não foi realizado diferenciando-se os graus de perda de audição. Devido ao período de aclimatização, ou seja, à gradual melhora nas habilidades auditivas (em especial, na compreensão da fala) decorrente da plasticidade do sistema auditivo, devida à reintrodução da estimulação auditiva a partir da amplificação( ${ }^{(13-15)}$, optou-se pela avaliação de novos usuários de próteses auditivas com no mínimo três meses de uso das próteses.

Todos os voluntários da pesquisa foram adaptados bilateralmente, com próteses auditivas de diferentes empresas, dos tipos retroauricular ou intra-aural, com processamento de sinal digital e amplificação do tipo não-linear (Wide Dynamic Range Compression - WDRC). Foram aplicados os questionários Hearing Handicap Inventory for Elderly (HHIE) no grupo formado por idosos, e Hearing Handicap Inventory for Adults (HHIA) no grupo formado por adultos, bem como o questionário Abbreviated Profile of Hearing Aid Benefit (APHAB) em ambos os grupos.

As aplicações dos questionários para a avaliação da restrição de participação em atividades de vida diária (HHIE ou HHIA), ou handicap auditivo, e das limitações de atividades impostas pela deficiência auditiva (APHAB) ocorreram em duas ocasiões: primeiramente, na ocasião da dispensação das próteses auditivas, quando o paciente foi orientado a responder aos questionários baseando-se em sua experiência sem o uso da amplificação; e, num segundo momento, considerando o uso das próteses, com no mínimo três meses de uso efetivo destas, respeitando o período de aclimatização. $\mathrm{O}$ uso diário das próteses auditivas foi considerado efetivo quando o paciente referiu ser igual ou superior a oito horas diárias. $\mathrm{O}$ uso foi de no mínimo oito e no máximo 14 horas.

Após o recebimento das próteses auditivas, bem como das orientações referentes à utilização, funcionamento e manutenção dos equipamentos, os voluntários foram solicitados a responderem os questionários HHIE ou HHIA, dependendo do grupo no qual foram incluídos, e ao APHAB, baseando-se em suas experiências sem o uso de amplificação sonora.

O questionário HHIE, proposto em $1982 \mathrm{com}$ a finalidade de avaliar os efeitos psicossociais da deficiência auditiva ${ }^{(16)} \mathrm{e}$ adaptado para o português em $1997^{(17)}$, é composto por 25 questões, das quais 13 se destinam à análise do impacto emocional causado pela deficiência auditiva e 12 à análise do impacto da referida perda no âmbito social/situacional na população idosa.

O questionário HHIE foi modificado em 1990, originando o HHIA ${ }^{(18)}$. Houve a substituição de três perguntas com a finalidade de analisar nos indivíduos adultos as limitações decorrentes do déficit auditivo no âmbito ocupacional e em atividades de lazer.

O questionário $\mathrm{APHAB}$, proposto em $1995^{(19)}$, é composto por 24 itens, os quais abrangem quatro subescalas: Facilidade de Comunicação (FC), Ambientes Reverberantes (RV), Ruído Ambiental (RA) e Aversão a Sons (AS).

Para a aplicação do questionário HHIE e HHIA, os vo- 
luntários foram instruídos a responderem "sim", "não" ou "às vezes", para cada uma das 24 questões, as quais foram apresentadas pela avaliadora.

Às respostas "sim", foram atribuídos 4 pontos; para as "às vezes", 2 pontos e para as respostas "não" não foram atribuídos pontos.

O valor da pontuação do HHIE e do HHIA pode variar de 0 a $100 \%$. Pontuações superiores a $42 \%$ indicam percepção severa ou significativa do handicap, pontuações de $18 \%$ a $42 \%$, percepção de leve a moderada e, pontuações inferiores a $16 \%$, não haver percepção da restrição de participação ${ }^{(17)}$.

Para a aplicação do questionário APHAB, que foi efetuada pela avaliadora, os voluntários foram instruídos a indicarem uma das sete opções de resposta para cada um dos vinte e quatro itens, sendo que a opção selecionada deveria corresponder à que mais se aproximava de seu cotidiano.

As opções de respostas, acima referidas, são "sempre", "quase sempre", "geralmente", "metade do tempo", "ocasionalmente", "raramente" e "nunca". O paciente seleciona uma destas opções, que está associada a uma porcentagem, a saber, $99 \%, 87 \%, 75 \%, 50 \%, 25 \%, 12 \%$, e $1 \%$, respectivamente ${ }^{(19)}$.

Após o período mínimo de três meses, os pacientes que fizeram uso efetivo das próteses auditivas (mínimo de oito horas por dia), ao retornarem à instituição para acompanhamento, foram solicitados a responderem, novamente, aos questionários de auto-avaliação; todavia, neste segundo momento, baseandose em suas experiências com o uso da amplificação sonora.

Para a verificação da redução das restrições de participação em atividades de vida diária, foram comparados os escores pré e pós-adaptação das próteses auditivas. É considerada melhora significativa na percepção do handicap, quando há redução de $36 \%$, se a aplicação do questionário utilizado foi realizada no formato papel e lápis, e de 19\%, se a aplicação ocorreu em forma de entrevista ${ }^{(20)}$.

Devido à baixa escolaridade da população atendida no setor em que foi realizado o estudo, foi adotado o procedimento da entrevista para a aplicação dos questionários com o intuito de garantir a compreensão do paciente quanto ao que lhe fora solicitado.
Para a verificação do benefício obtido com o uso de próteses auditivas por meio do APHAB, é possível realizar a análise por subescala ou global, considerando conjuntamente as subescalas Facilidade de Comunicação, Ambientes Reverberantes e Ruído Ambiental.

Ao analisar uma subescala específica, considera-se uma diferença significativa quando há redução, entre as condições sem e com prótese auditiva, de 22 pontos para as subescalas Facilidade de Comunicação, Ambientes Reverberantes e Ruído Ambiental, e de 31 pontos para a subescala Aversão a Sons ${ }^{(21)}$.

Ao realizar a análise de forma global, considera-se uma diferença significativa quando há redução de pelo menos cinco pontos em cada uma das três subescalas consideradas entre as condições com e sem prótese auditiva. Cabe ressaltar que considerar significante uma diferença de dez pontos entre as condições referidas representa um real benefício ${ }^{(21)}$.

As respostas do questionário $\mathrm{APHAB}$ foram quantificadas por um programa disponibilizado por uma empresa de aparelhos auditivos em seu software de programação das próteses auditivas.

Subsequentemente à coleta dos dados, os mesmos foram submetidos à análise estatística pertinente. Os testes estatísticos utilizados foram o teste de Wilcoxon, a fim de comparar, em cada um dos grupos, o resultado entre primeira e segunda avaliações, e o teste de Correlação de Spearman, para comparar as resultados do HHIE com os do APHAB e os resultados do HHIA com o APHAB. O nível de significância adotado para as análises realizadas foi de $0,05(5 \%)$.

\section{RESULTADOS}

Inicialmente, investigou-se se a utilização das próteses auditivas determinou mudanças na percepção dos idosos (GI) quanto à restrição de participação em atividades de vida diária e às dificuldades impostas pela deficiência auditiva em ambientes favoráveis à comunicação, reverberantes, ruidosos e em presença de sons aversivos (Tabela 1).

Os resultados estatísticos (Teste de Wilcoxon) revelaram que houve diferenças significantes entre os escores do ques-

Tabela 1. Medidas descritivas dos escores dos questionários HHIE e APHAB (FC, RV, RA, AS) nos dois momentos de avaliação e resultados do teste de Wilcoxon para o grupo de idosos (GI)

\begin{tabular}{|c|c|c|c|c|c|c|c|c|c|}
\hline Quest & & Média & Mediana & DP & Q1 & Q3 & $\mathrm{n}$ & IC & Valor de $p$ \\
\hline \multirow{2}{*}{ HHIE } & $1^{a}$ avaliação & 41,93 & 42 & 24,26 & 21 & 8 & 27 & 9,15 & \multirow{2}{*}{$<0,001^{\star}$} \\
\hline & $2^{\mathrm{a}}$ avaliação & 15,78 & 8 & 18,03 & 4 & 0 & 27 & 6,8 & \\
\hline \multirow{2}{*}{$\mathrm{FC}$} & $1^{a}$ avaliação & 66,78 & 68 & 17,39 & 51 & 37 & 27 & 6,56 & \multirow{2}{*}{$<0,001^{*}$} \\
\hline & $2^{\mathrm{a}}$ avaliação & 38,96 & 35 & 15,43 & 27 & 13 & 27 & 5,82 & \\
\hline \multirow{2}{*}{$\mathrm{RV}$} & $1^{a}$ avaliação & 60,85 & 56 & 17,87 & 50 & 35 & 27 & 6,74 & \multirow{2}{*}{$<0,001^{*}$} \\
\hline & $2^{\mathrm{a}}$ avaliação & 32,67 & 33 & 9,72 & 25 & 12 & 27 & 3,67 & \\
\hline \multirow{2}{*}{ RA } & $1^{\mathrm{a}}$ avaliação & 52,67 & 54 & 22,78 & 36 & 4 & 27 & 8,59 & \multirow{2}{*}{$<0,001^{\star}$} \\
\hline & $2^{\mathrm{a}}$ avaliação & 30,19 & 29 & 16,04 & 17 & 1 & 27 & 6,05 & \\
\hline \multirow{2}{*}{ AS } & $1^{a}$ avaliação & 25,7 & 6 & 29,21 & 1 & 1 & 27 & 11,02 & \multirow{2}{*}{0,754} \\
\hline & $2^{\mathrm{a}}$ avaliação & 22,85 & 13 & 23,04 & 3 & 1 & 27 & 8,69 & \\
\hline
\end{tabular}

* Valores significativos $(p<0,05)$ - Teste de Wilcoxon

Legenda: $\mathrm{DP}=$ desvio padrão; $\mathrm{HHIE}=$ Hearing Handicap Inventory for Elderly; $\mathrm{FC}=$ facilidade de comunicação; $\mathrm{RV}=$ ambientes reverberantes; $\mathrm{RA}=$ ruído ambiental; AS = aversão a sons; $Q 1$ = primeiro quartil; $Q 3=$ terceiro quartil; IC = intervalo de confiança 
Tabela 2. Medidas descritivas dos escores dos questionários HHIA e APHAB (FC, RV, RA, AS) nos dois momentos de avaliação e resultados do teste de Wilcoxon para o grupo de adultos (GA)

\begin{tabular}{|c|c|c|c|c|c|c|c|c|c|}
\hline Questic & & Média & Mediana & DP & Q1 & Q3 & $\mathrm{n}$ & IC & Valor de $p$ \\
\hline \multirow{2}{*}{$\mathrm{HHIA}$} & $1^{a}$ Avaliação & 59,53 & 60 & 20,11 & 50 & 24 & 17 & 9,56 & \multirow{2}{*}{$<0,001^{*}$} \\
\hline & $2^{a}$ Avaliação & 26,94 & 22 & 19,58 & 16 & 0 & 17 & 9,31 & \\
\hline \multirow{2}{*}{ FC } & $1^{a}$ Avaliação & 66,94 & 64 & 13,7 & 56 & 43 & 17 & 6,51 & \multirow{2}{*}{$<0,001^{*}$} \\
\hline & $2^{\mathrm{a}}$ Avaliação & 29,06 & 25 & 17,25 & 19 & 2 & 17 & 8,2 & \\
\hline \multirow{2}{*}{ RV } & $1^{a}$ Avaliação & 57,71 & 58 & 19,18 & 50 & 19 & 17 & 9,12 & \multirow{2}{*}{$0,001^{*}$} \\
\hline & $2^{a}$ Avaliação & 28,65 & 25 & 18,79 & 18 & 1 & 17 & 8,93 & \\
\hline \multirow{2}{*}{ RA } & $1^{a}$ Avaliação & 58,88 & 64 & 18,68 & 43 & 29 & 17 & 8,88 & \multirow{2}{*}{$<0,001^{*}$} \\
\hline & $2^{a}$ Avaliação & 33,35 & 29 & 19,35 & 17 & 4 & 17 & 9,2 & \\
\hline \multirow{2}{*}{ AS } & $1^{a}$ Avaliação & 35,29 & 31 & 21,64 & 23 & 1 & 17 & 10,28 & \multirow{2}{*}{0,469} \\
\hline & $2^{a}$ Avaliação & 33,59 & 23 & 23,62 & 16 & 1 & 17 & 11,23 & \\
\hline
\end{tabular}

* Valores significativos $(p<0,05)$ - Teste de Wilcoxon

Legenda: DP = desvio padrão; HHIA = Hearing Handicap Inventory for Adults; FC = facilidade de comunicação; RV = ambientes reverberantes; RA = ruído ambiental; AS = aversão a sons; $\mathrm{Q1}$ = primeiro quartil; Q3 = terceiro quartil; IC = intervalo de confiança

tionário HHIE e das subescalas FC, RV e RA do questionário APHAB obtidos na primeira e segunda avaliação. Não houve diferença significativa entre os escores da subescala AS obtidos na primeira e na segunda avaliação.

A seguir, a mesma análise foi realizada no grupo de adultos (GA) (Tabela 2).

Assim como no Grupo de Idosos, a análise (Teste de Wilcoxon) revelou que houve diferenças significantes entre os escores do questionário HHIA e das subescalas FC, RV e RA do questionário APHAB obtidos na primeira e segunda avaliação. Não houve diferença estatística entre os escores da subescala AS obtidos na primeira e na segunda avaliação.

Por fim, foi investigado se houve correlação entre a redução da restrição de participação mensurada pelos questionários HHIE ou HHIA e a redução das limitações de atividades investigadas pelo questionário APHAB (benefício) (Tabelas 3 e 4 , respectivamente).

Tabela 3. Resultados do teste de Correlação de Spearman entre a redução das restrições de participação em atividades de vida diária e o benefício nas subescalas do APHAB no grupo de idosos

\begin{tabular}{llccccc}
\hline Questionário & HHIE & FC & RV & RA & AS \\
\hline \multirow{2}{*}{ HHIE } & Correlação & - & & & & \\
& Valor de $p$ & 1,000 & & & & \\
\multirow{2}{*}{ FC } & Correlação & 38,8 & & & & \\
& Valor de $p$ & $0,046^{*}$ & & & & \\
\multirow{2}{*}{ RV } & Correlação & 28,2 & 29,2 & & & \\
& Valor de p & 0,154 & 0,139 & & & \\
RA & Correlação & 29,9 & 43,2 & 43,9 & & \\
& Valor de p & 0,130 & $0,024^{*}$ & $0,022^{*}$ & & \\
\multirow{2}{*}{ AS } & Correlação & 5,1 & 3,4 & 30,5 & 22,8 & - \\
& Valor de p & 0,800 & 0,867 & 0,122 & 0,253 & 1,000 \\
\hline
\end{tabular}

*Valores significativos $(p<0,05)$ - Correlação de Spearman Valores de correlação expressos em \%

Legenda: $\mathrm{HHIE}=$ Hearing Handicap Inventory for Elderly; $\mathrm{FC}=$ facilidade de comunicação; $\mathrm{RV}$ = ambientes reverberantes; $\mathrm{RA}$ = ruído ambiental; $\mathrm{AS}$ = aversão a sons
Tabela 4. Resultados do teste de Correlação de Spearman entre a redução das restrições de participação em atividades de vida diária e o benefício nas subescalas do APHAB no grupo de adultos

\begin{tabular}{lllllll}
\hline Questionário & HHIA & FC & RV & RA & AS \\
\hline \multirow{2}{*}{ HHIA } & Correlação & - & & & & \\
& Valor de p & 1,000 & & & & \\
\multirow{2}{*}{ FC } & Correlação & 47,5 & & & & \\
& Valor de p & $0,054 \#$ & & & & \\
\multirow{2}{*}{ RV } & Correlação & 40,0 & 35,2 & & & \\
& Valor de p & 0,111 & 0,165 & & & \\
\multirow{2}{*}{ RA } & Correlação & 49,4 & 53,5 & 76,4 & & \\
& Valor de p & $0,044^{*}$ & $0,027^{*}$ & $<0,001^{*}$ & \\
\multirow{2}{*}{ AS } & Correlação & 8,4 & 7,8 & 29,6 & 31,3 & - \\
& Valor de p & 0,748 & 0,766 & 0,248 & 0,221 & 1,000 \\
\hline
\end{tabular}

*Valores significativos $(p<0,05)$ - Correlação de Spearman

\# Valores com tendência à significância estatística

Valores de correlação expressos em \%

Legenda: HHIA = Hearing Handicap Inventory for Adults; FC = facilidade de comunicação; $\mathrm{RV}=$ ambientes reverberantes; $\mathrm{RA}=$ ruído ambiental; $\mathrm{AS}=$ aversão a sons

Quanto ao GI, os resultados estatísticos (Correlação de Spearman) revelaram que houve uma correlação positiva entre a redução das restrições de participação em atividades de vida diária (HHIE) e o benefício na subescala FC do APHAB e entre o benefício na subescala RA com o benefício das subescalas FC e RV. Tais achados sugerem que quanto maior o benefício na subescala FC, maior a redução das restrições de participação em atividades de vida diária e, quanto maior o benefício em RA, maior o benefício em FC e RV.

Quanto ao GA, os resultados demonstraram uma correlação positiva entre o benefício da subescala RA do questionário APHAB e a redução das restrições de participação em atividades de vida diária e entre o benefício em RA e as subescalas FC e RV. Tais achados sugerem que quanto maior o benefício na subescala RA maior a redução das restrições de participação e o benefício nas subescalas FC e RV. 


\section{DISCUSSÃO}

Este estudo revelou que, em ambos os grupos, nas subescalas FC, RV e RA do questionário APHAB, as diferenças entre a primeira e a segunda avaliação foram significativas, demonstrando haver uma importante redução das dificuldades auditivas enfrentadas pelos idosos e adultos em ambientes com facilidade de comunicação, reverberantes e com ruído de fundo, após um período mínimo de 12 semanas de utilização de próteses auditivas (Tabelas 1 e 2). Os resultados sugerem que uma adequada adaptação de próteses auditivas, que compreende desde as orientações e programação realizadas ao uso efetivo destas pelo paciente, é capaz de gerar benefícios auditivos em diferentes ambientes acústicos, minimizando as dificuldades geradas pela perda de audição neurossensorial. Tais achados concordam com os resultados encontrados em diversos estudos, os quais também encontraram diferenças significativas entre as condições com e sem próteses auditi$\operatorname{vas}^{(6,22,23)}$.

Quanto à aplicação do APHAB, sabe-se que uma diferença de dez pontos entre as condições com e sem prótese auditiva representa um real benefício ${ }^{(21)}$.

O benefício no GI foi, em média, $27,82 \%, 28,18 \%$ e $22,48 \%$, nas subescalas FC, RV e RA, respectivamente, enquanto que no GA foi, em média, 37,88\%, 29,06\% e 25,53\%, nas subescalas FC, RV e RA, respectivamente. Quanto à subescala Aversão a Sons, o benefício médio foi de $2,85 \%$ nos idosos e de $1,70 \%$ nos adultos, não sendo observada diferença significativa entre as condições com e sem próteses auditivas em nenhum dos grupos estudados, embora todos pacientes utilizassem próteses auditivas com circuito WDRC que, pela não linearidade do processamento do sinal, possibilitam minimizar os problemas com sons considerados intensos e desconfortáveis.

Segundo a autora do questionário APHAB esta última subescala não é bem compreendida, havendo a necessidade de realização de mais pesquisas antes de agrupar a subescala relacionada a sons aversivos às demais ${ }^{(21)}$.

A melhora observada nos dois grupos na segunda avaliação é plenamente justificada pela redução das dificuldades auditivas com o uso da amplificação. Já, o maior benefício observado no grupo de adultos, poderia ser justificado pela maior exposição destes às atividades sociais e de trabalho, o que possibilitaria maior percepção do desempenho comunicativo que os idosos.

Já a análise da redução das restrições de participação em atividades de vida diária revelou que esta foi significativa após um período de utilização da amplificação sonora, visto que, no grupo de idosos, tais restrições passaram de 41,35\% para $15,78 \%$, e no grupo de adultos, passaram de 59,53\% para $26,94 \%$. Estes resultados concordam com os achados de outros pesquisadores $^{(15,24)}$.

A redução das restrições de participação nas atividades de vida diária observada por meio da aplicação do HHIE e HHIA reflete o benefício verificado pela aplicação do questionário APHAB. Ou seja, o questionário APHAB mostrou um benefício significativo nas situações de facilidade de comunicação, de comunicação em ambiente reverberante e com ruído ambiental por meio do uso das próteses auditivas, o que, provavelmente, facilitou o processo de comunicativo do paciente nas mais diversas situações, levando consequentemente a uma redução das restrições de participação nas atividades do dia a dia.

Sabe-se que, na análise da redução das restrições de participação em atividades de vida diária, uma mudança de $19 \%$ no escore dos questionários entre as condições sem e com próteses auditivas é considerada significativa quando a aplicação do instrumento ocorre em forma de entrevista ${ }^{(20)}$, como o realizado neste estudo.

É esperada uma redução das restrições de participação em atividades de vida diária com a utilização de amplificação sonora, visto que o objetivo da reabilitação auditiva é minimizar os efeitos da perda de audição, tanto os auditivos, como a dificuldade de detecção e compreensão do sinal acústico, quanto os emocionais e sociais.

O estudo da correlação entre os questionários HHIE e APHAB (Tabela 3) revelou correlação positiva entre os resultados do HHIE e os da subescala FC do APHAB, entre os resultados das subescalas FC e RA, bem como entre os resultados das subescalas RA e RV. Portanto, quanto maior o benefício referido em ambientes onde há facilidade de comunicação, maior a redução das restrições de participação em atividades de vida diária e o benefício referido em ambientes ruidosos, e quanto maior o benefício em ambientes com ruído de fundo, maior o benefício em ambientes reverberantes. Tais resultados indicaram que quanto menor é a dificuldade auditiva em ambientes silenciosos, menor é a restrição de participação enfrentada por idosos, e menor a limitação auditiva em ambientes ruidosos. Os resultados revelaram também que, quanto menor é a limitação auditiva em ambientes ruidosos, menor é a limitação em ambientes com reverberação, que, assim como os ruidosos, apresenta uma considerável distorção do sinal acústico.

A análise da correlação entre questionários HHIA e APHAB (Tabela 4) apontou correlação positiva entre os escores obtidos na subescala RA com os obtidos nas subescalas FC e RV do questionário APHAB e dos escores do HHIA. Portanto, quanto maior o benefício referido em ambientes ruidosos (RA), maior a redução das restrições de participação em atividades de vida diária no HHIA e maior o benefício em ambientes favoráveis à comunicação (FC) e em ambientes com reverberação (RV). Estes resultados demonstraram que para os adultos, quanto menor é a limitação auditiva em ambientes com ruído ambiental (RA), nos quais há uma distorção da fala, devido ao ruído de fundo, menor é a limitação em ambientes que favoreçam a comunicação (FC), ou seja, ambientes silenciosos, e também em situações mais adversas para a comunicação como os ambientes reverberantes (RV). Além disto, quanto menor é a limitação auditiva em ambientes com ruído de fundo (RA), menor é a restrição de participação dos adultos com deficiência auditiva, apontada pelos resultados do questionário HHIA.

Não foram encontrados na literatura achados quanto à correlação dos questionários HHIE ou HHIA com o questionário $\mathrm{APHAB}$ que pudessem ser comparados aos achados do presente estudo; portanto, faz-se necessária a realização de mais estudos que visem comparar tais questionários, os quais 
são os mais utilizados na prática clínica.

Para a utilização qualquer instrumento de avaliação na prática clínica, é necessário o conhecimento não apenas sobre a forma como deve ser aplicado, mas também sobre quais as informações que podem ser extraídas de tal aplicação.

Independentemente do instrumento selecionado para verificar o benefício da amplificação, é importante ao fonoaudiólogo não visualizar apenas os escores obtidos pelo paciente com e sem o uso da prótese auditiva, mas quais informações sobre a vida do paciente tal instrumento pode fornecer.

Com a realização da presente pesquisa, não se pretendeu recomendar o uso de um questionário específico utilizado (HHIE ou HHIA e APHAB) ou de ambos, mas mostrar que, ao optar por avaliar as limitações auditivas com e sem a amplificação sonora, o profissional pode inferir quais os impactos destas na participação social e atividades de vida diária e vice-versa.

Por fim, vale salientar que durante a realização da pesquisa, foram encontradas dificuldades na aplicação dos questionários quanto à compreensão das perguntas pelos pacientes, principalmente com relação aos questionamentos sobre as dificuldades enfrentadas em situações pouco experienciadas por estes.

Este estudo revela a importância de utilizar sistematicamente pelo menos um questionário de auto-avaliação na prática clínica (pública ou privada), uma vez que isso pode auxiliar o fonoaudiólogo na obtenção de informações importantes sobre as dificuldades residuais, satisfação e qualidade de vida do usuário e, nos acompanhamentos, auxiliar nos ajustes finos e no aconselhamento.

\section{CONCLUSÃO}

Idosos e adultos com deficiência auditiva neurossensorial percebem redução das limitações auditivas em ambientes acústicos favoráveis à comunicação, reverberantes e ruidosos, bem como da restrição de participação em atividades de vida diária com o uso de próteses auditivas.

Há correlação positiva entre a redução da restrição de participação em atividades de vida diária e o benefício obtido em ambientes acusticamente favoráveis à comunicação por idosos usuários de próteses auditivas.

Há correlação positiva entre a redução da restrição de participação em atividades de vida diária e o benefício obtido em ambientes ruidosos por usuários adultos de próteses auditivas.

\section{AGRADECIMENTOS}

À Fundação de Amparo à Pesquisa do Estado de São Paulo (FAPESP) pelo apoio concedido para a realização desta pesquisa por meio da Bolsa de Iniciação Científica, sob número 2008/57051-8.

\begin{abstract}
Purpose: To study the benefit obtained by the use of hearing aids in environments acoustically favorable to communication, reverberant, noisy and with aversive sounds, and to correlate the benefit with the handicap of adults and elderly with hearing loss. Methods: Participants were 42 individuals attended at the institution where the study was carried. They answered the questionnaires Hearing Handicap Inventory for Elderly or for Adults, and Abbreviated Profile of Hearing Aid Benefit both when they received the hearing aids and after three months of its effective use. The participation constraints, hearing difficulties and the benefit brought by the use of hearing aids were studied. The results were statistically analyzed. Results: There were differences between the scores from the questionnaires Hearing Handicap Inventory for Elderly and Hearing Handicap Inventory for Adults and the subscales Ease of Communication, Reverberation and Background Noise from the Abbreviated Profile of Hearing Aid Benefit, comparing the conditions with and without hearing aids. There was a positive correlation between the benefit measured by the subscale Ease of Communication, for the elderly subjects, and Background Noise, for the adult subjects, with decrease of the participation constraint. Conclusion: Adult and elderly subjects showed a reduction of handicap in daily activities with the use of hearing aids. The bigger the benefit obtained in the subscale Ease of Communication for the elderly and in the subscale Background Noise for the adults, the greater the handicap reduction.
\end{abstract}

Keywords: Rehabilitation of hearing impaired; Hearing aids; Hearing loss, sensorineural; Hearing; Adult; Aged

\title{
REFERÊNCIAS
}

1. Goulart BN, Chiari BM. Avaliação clínica fonoaudiológica, integralidade e humanização: perspectivas gerais e contribuições para reflexão. Rev Soc Bras Fonoaudiol. 2007;12(4):335-40.

2. Miranda EC, Calais LL, Vieira EP, Carvalho LM, Borges AC, Iório MC. Dificuldades e benefícios com o uso de prótese auditiva: percepção do idoso e sua família. Rev Soc Bras Fonoaudiol. 2008;13(2):166-72.

3. Fialho IM, Bortoli D, Mendonça GG, Pagnosim DF, Scholze AS. Percepção de idosos sobre o uso de AASI concedido pelo Sistema Único de Saúde. Rev CEFAC. 2009;11(2):338-44.
4. Macedo LS, Pupo AC, Balieiro CR. Aplicabilidade dos questionários de auto-avaliação em adultos e idosos com deficiência auditiva. Distúrb Comun. 2006;18(1):19-25.

5. Russo IC, Almeida K, Freire KG. Seleção e adaptação da prótese auditiva para o idoso. In: Almeida K, Iório MC. Próteses auditivas: fundamentos teóricos e aplicações clínicas. 2a ed. São Paulo: Lovise; c2003. p. 385-410.

6. Freitas CD, Costa MJ. Processo de adaptação de próteses auditivas em usuários atendidos em uma instituição pública federal: parte 
II: resultados dos questionários de auto-avaliação. Rev Bras Otorrinolaringol. 2007;73(5):660-70.

7. Rosis AC, Souza MR, Iório MC. Questionário Hearing Handicap Inventory for the Elderly - Screening version (HHIE-S): estudo da sensibilidade e especificidade. Rev Soc Bras Fonoaudiol. 2009;14(3):339-45.

8. Costa MH, Sampaio AL, Oliveira CA. Avaliação do benefício da prótese auditiva digital e da percepção da desvantagem auditiva ou "handicap" em idosos não institucionalizados. Arq Int Otorrinolaringol. 2007;11(2):159-68.

9. Teixeira AR, Almeida LG, Jotz GP, De Barba MC. Qualidade de vida de adultos e idosos pós adaptação de próteses auditivas. Rev Soc Bras Fonoaudiol. 2008;13(4):357-61.

10. Rosa MR, Dante G, Ribas A. Programa de orientação a usuários de prótese auditiva e questionários de auto-avaliação: importantes instrumentos para uma adaptação auditiva efetiva. Arq Int Otorrinolaringol. 2006;10(3):220-7.

11. Cox RM, Alexander GC. Measuring satisfaction with Amplification in Daily Life: the SADL scale. Ear Hear. 1999;20(4):306-20.

12. Cox RM, Alexander GC, Beyer CM. Norms for the international outcome inventory for hearing aids. J Am Acad Audiol. 2003;14(8):40313.

13. Prates LP, Iório MC. Aclimatização: estudo do reconhecimento de fala em usuários de próteses auditivas. Pró-Fono. 2006;18(3):259-66.

14. Miranda EC, Andrade AN, Gil D, Iório MC. A efetividade do treinamento auditivo formal em idosos usuários de próteses auditivas no período de aclimatização. Rev Soc Bras Fonoaudiol. 2007;12(4):316-21.
15. Amorim RM, Almeida K. Estudo do benefício e da aclimatização em novos usuários de próteses auditivas. Pró-Fono. 2007;19(1):39-48.

16. Ventry IM, Weinstein BE. The hearing handicap inventory for elderly: a new tool. Ear Hear. 1982;3(3):128-34.

17. Wieselberg MB. A auto-avaliação do handicap em idosos portadores de deficiência auditiva: o uso do HHIE [dissertação]. São Paulo: Pontifícia Universidade Católica de São Paulo; 1997.

18. Newman CW, Weinstein BE, Jacobson GP, Hug GA. The hearing handicap inventory for adults: psychometric adequacy and audiometric correlates. Ear Hear. 1990;11(6):430-3.

19. Cox RM, Alexander GC. The abbreviated profile of hearing aid benefit. Ear Hear. 1995;16(2):176-83.

20. Kricos PB, Lesner SA. Evaluating the success of adult audiologic rehabilitation support programs. Semin Hear. 2000;21(3):267-80.

21. Cox RM. Administration and application of the APHAB. Hear J. 1997;50(4):32-48.

22. Assayag FH, Russo IC. Avaliação subjetiva do benefício e dos efeitos proporcionados pelo uso de amplificação sonora em indivíduos idosos. Distúrb Comun. 2006;18(3):383-90.

23. Gordo A, Scharlach RC, Iório MC. Avaliação do benefício de próteses auditivas com diferentes tipos de processamento do sinal por meio da aplicação do questionário APHAB. Distúrb Comun. 2005;17(2):191202.

24. Buzo BC, Ubrig MT, Novaes BC. Adaptação de aparelho de amplificação sonora individual: relações entre a auto-percepção do handicap auditivo e a avaliação da percepção de fala. Distúrb Comun. 2004;16(1):17-25. 